\title{
An Efficient Transformation of Cyclic Ene-carbamates into $\omega-(N-$ Formylamino)carboxylic Acids by Ruthenium Tetroxide Oxidation
}

\author{
Mamoru Kaname, Shigeyuki YoshifuJI, and Haruki SaSHIDA* \\ Faculty of Pharmaceutical Sciences, Hokuriku University; Kanagawa-machi, Kanazawa 920-1181, Japan. \\ Received May 20, 2008; accepted July 1, 2008; published online July 2, 2008
}

The ruthenium tetroxide $\left(\mathrm{RuO}_{4}\right)$ oxidation of cyclic ene-carbamates resulted in the endo-cyclic carbon-carbon double bond cleavage to afford the corresponding $\omega$-( $N$-formylamino)carboxylic acids in good yields. Substituted cyclic ene-carbamates derived from (3R)-3-hydroxypiperidine hydrochloride were converted into the $N$-Boc 4-aminobutyric acids by utilization of the $\mathrm{RuO}_{4}$ oxidation as the key step, which were further transformed into (3R)-4-amino-3-hydroxybutyric acid, an important key intermediate for the synthesis of L-carnitine.

Key words $\mathrm{RuO}_{4}$ oxidation; cyclic ene-carbamate; endo-cyclic carbon-carbon double bond cleavage; L-carnitine

The high-valent metal-promoted oxidation of a carboncarbon double $(\mathrm{C}=\mathrm{C})$ bond with cleavage is a promising synthetic route to the corresponding carbonyl compounds. This chemistry has been extensively studied. ${ }^{1)}$ Ruthenium tetroxide $\left(\mathrm{RuO}_{4}\right)$ is well known as a highly effective oxidant, $^{2-4)}$ and the oxidative cleavage of simple $\mathrm{C}=\mathrm{C}$ bonds is one of the most common reactions between $\mathrm{RuO}_{4}{ }^{2)}$ and alkenes. However, only a low effort regarding the oxidative cleavage of the $\mathrm{C}=\mathrm{C}$ double bond to the corresponding carbonyl compounds in enol ethers, enol esters and enamines has been made to date. To the best of our knowledge, there are only two papers. The first example of this type of reaction was described by Desai and co-workers, ${ }^{5)}$ who reported one case that attempted the cleavage of a $\mathrm{C}=\mathrm{C}$ bond in steroidal enamines. Torii and co-workers ${ }^{6}$ revealed the procedure for the $\mathrm{RuO}_{4}$ oxidative cleavage of enolic olefins including two six-membered nitrogen-containing heterocyles that afforded the carbonyl compounds under the conditions using the substrate in a suspension of $\mathrm{CCl}_{4}$ and $\mathrm{H}_{2} \mathrm{O}$ in the presence of $\mathrm{RuO}_{2} \cdot x \mathrm{H}_{2} \mathrm{O}$ and $\mathrm{NaIO}_{4}$.

On the other hand, we have been working on the transformation of cyclic $^{7-9)}$ and acyclic ${ }^{10-12)} N$-acyl amines into the corresponding lactams and imides including the natural products ${ }^{13-17)}$ by the $\mathrm{RuO}_{4}$ oxidation. The double layer oxidation method using a catalytic amount of $\mathrm{RuO}_{2}$ hydrate, an excess of a $\mathrm{NaIO}_{4}$ aqueous solution and ethyl acetate system was established for these purposes in our laboratory. In addition, it was recently reported that the single layer $\mathrm{RuO}_{4}$ oxidation $^{18)}$ of cyclic $\mathrm{N}$-acyl amines using a tert-butanol and $\mathrm{NaIO}_{4}$ aqueous solution system gave the ring opened $\omega$ amino acids (Chart 1). We now report that the simple $\mathrm{RuO}_{4}$ oxidation of various cyclic ene-carbamates including the optically active one into the corresponding $\omega$-( $N$-formylamino)carboxylic acids and the formal synthesis of L-carnitine as the utilization of this $\mathrm{RuO}_{4}$ oxidation.

\section{Results and Discussion}

The substrates, five-, six- and seven-membered cyclic ene-

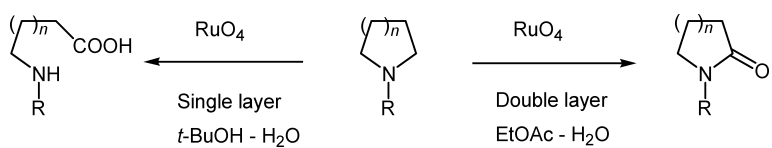

Chart 1 carbamates (1a, 2a, 3a), were prepared from the corresponding $\mathrm{N}$-acyl amines via the $\mathrm{N}$-acyl lactams in good yields by the reported method. ${ }^{19)}$ We have previously tested the utility of the urethane type $\mathrm{N}$-protecting groups, ${ }^{9)}$ benzyloxycarbonyl (Z), $p$-nitrobenzyloxycarbonyl (PNZ), trichloroethoxycarbonyl (Troc) and tert-butoxycarbonyl (Boc) groups for the $\mathrm{RuO}_{4}$ oxidation of the cyclic amines, and found the following three points: (1) the $\mathrm{Z}$ group is decomposed by $\mathrm{RuO}_{4}$ that produced a low yield of products, (2) the PNZ and Troc groups generally require a longer reaction until the starting materials disappear, and (3) the Boc group is stable, accelerates the oxidation, and affords the products in high yields. In addition, the Boc group can be easily removed from the $N$ protected amines by common procedures. Thus, we selected the Boc group as the $\mathrm{N}$-protecting group for this $\mathrm{RuO}_{4}$ oxidation.

$\mathrm{RuO}_{4}$ Oxidation of Ene-carbamates The oxidation of $N$-tert-butoxycarbonyl (Boc) pyrroline (1a) with $\mathrm{RuO}_{4}$ using the established conventional double layer system of ethyl acetate and $10 \% \mathrm{NaIO}_{4}$ as a co-oxidant at $0{ }^{\circ} \mathrm{C}$ resulted in the successful endo-cyclic $\mathrm{C}=\mathrm{C}$ bond cleavage to afford the desired $\omega$-( $N$-formylamino)propanoic acid (1b) in $86 \%$ yield (Table 1, entry 1). The results obtained using several cyclic ene-carbamates are also summarized in Table 1. Six- (2a) and seven-membered cyclic ene-carbamates (3a) were also converted to the corresponding $\omega$-( $N$-formylamino) carboxylic acid (2b, 3b) in 78 and $90 \%$ yields under the same conditions, respectively (entries 2,3 ). Next, this $\mathrm{RuO}_{4}$ oxidation of the ene-carbamates was employed using the optically active amine frameworks. The five-membered $N$-Boc enamine $(\mathbf{4 a})^{20-22)}$ having the methyl ester moiety at the $\mathrm{C}-2$ position was oxidized under similar conditions to afford the optically active $N$-formylamino carboxylic acid (4b) in $87 \%$ yield without any loss of chirality of the starting enamine $4 a$ (entry 4). A similar $\mathrm{C}=\mathrm{C}$ bond cleavage oxidation of the optically active six-membered enamine $\mathbf{5 a}^{23)}$ also proceeded to give the $N$-formyl derivative $\mathbf{5 b}$ in $74 \%$ yield (entry 5). This oxidation was also successfully applied to the transformation of the phosphonic diester. The $N$-Boc five-membered aminophosphonic acid diester (6a) was similarly converted into the $\omega$-( $N$-formylamino)carboxylic acids having a phosphonic diester moiety in $80 \%$ yield (entry 6 ).

Synthesis of L-Carnitine Finally, we describe the formal asymmetric synthesis of L-carnitine from the commercially 
available $(R)$-hydroxypiperidine hydrochloride as the starting material by using the $\mathrm{RuO}_{4}$ oxidation of both the $N$-acyl cyclic amines and enamines as two of the key steps (Chart 2). In recent years, carnitine and its analogues have attracted much attention because they have important biological activities. $^{24,25)}$ Few synthetic studies ${ }^{26)}$ including asymmetric syntheses, the utilization of chiral starting materials, chemical resolution, enzymatic or microbial techniques, etc., have been reported. The acetylation of tert-butyl (3R)-3-hydroxypiperidine-1-carboxylate $(7),{ }^{27)}$ prepared from $(R)$-hydroxypiperidine hydrochloride, with $\mathrm{Ac}_{2} \mathrm{O}$ in pyridine gave the 3acetyl derivative (8a) in $96 \%$ yield. 3-(tert-Butyldimethylsilyloxy)piperidine (8b) was obtained by the reaction of $\mathbf{7}$ and tert-butyldimethylsilyl chloride and imidazole in dry DMF in $97 \%$ yield. The first $\mathrm{RuO}_{4}$ oxidation of the $\mathrm{O}$-protected $\mathrm{N}$ Boc piperidines $(\mathbf{8 a}, \mathbf{8 b})$ resulted in the carbonylation at the

Table 1. $\mathrm{RuO}_{4}$ Oxidation of Cyclic Ene-carbamates

Entry

C-6 position to give the desired 5-acetoxypiperidones (9a) and 5-silyloxypiperidone (9b), together with the C-2 oxidative compounds (10a, 10b) in the yields shown in Chart 2. The 5-acetoxy (5-silyloxy)piperidones (9) and 3-acetoxy(3silyloxy) derivatives (10) could be separated by silica gel chromatography, and both structures of the products were determined by satisfactory spectroscopic (MS, IR, ${ }^{1} \mathrm{H}$ - and ${ }^{13} \mathrm{C}$ NMR) measurements. In the case of the former $9 \mathbf{a}$, the $5-\mathrm{H}$ signal appeared at $\delta 5.21$ as a multiplet in the ${ }^{1} \mathrm{H}-\mathrm{NMR}$. The $3-\mathrm{H}$ signal of the latter 10a was observed at $\delta 5.31$ as double-doublets in the lower field due to the deshielding effect of the carbonyl group at the $\mathrm{C}-2$ position. The major compounds 9 were converted into the corresponding ene-carbamates (11) by the reported method, ${ }^{19)}$ which was reduction of the lactam carbamate with Super-Hydride, followed by in situ dehydration with trifluoroacetic anhydride (TFAA) and diisopropylethylamine (DIPEA). The 3-acetoxy (11a) and 3-silyloxy-1,2,3,4-tetrahydropyridines (11b) were obtained from the corresponding lactams (9) in 71 and $82 \%$ yields, respectively. Next, the second $\mathrm{RuO}_{4}$ oxidation of the ene-carbamates (11) was carried out. The $\mathrm{RuO}_{4}$ oxidation for the endocyclic $\mathrm{C}=\mathrm{C}$ bond cleavage of the tetrahydropyridines (11) under our established conventional double layer system gave the desired ring-opening $\mathrm{N}$-formylaminocarboxylic acids (12a, 12b) in 80 and $78 \%$ yields. The hydroxylation of the 3-acetate (12a) with $2 \mathrm{M} \mathrm{HCl}$, followed by the desalting with ion-exchange chromatography (Dowex $50 \mathrm{~W} \times 8,2 \%$ $\left.\mathrm{NH}_{4} \mathrm{OH}\right)$ produced the free (3R)-4-amino-3-hydroxybutyric acid (13) in 80\% yield. Similarly, the free amino acid (13) was obtained from the 3-silyloxy derivative (12b) in $85 \%$ yield. 13 was identical to the authentic sample, ${ }^{28)}$ and was the key compound for the formal synthesis of L-carnitine (15).

In conclusion, $\mathrm{RuO}_{4}$ oxidized the carbon-carbon double bond of several cyclic ene-carbamates to give the $\omega-(N-$ formylamino)carboxylic acids in good to high yields. This oxidation could be successfully applied to the optically active tetrahydropyridines having an oxy-functional group at the C3 position giving the $N$-formylaminocarboxylic acids, which were converted into (3R)-4-amino-3-hydroxybutyric acid, an important key intermediate for the synthesis of L-carnitine. Thus, the formal asymmetric synthesis of L-carnitine was ac-

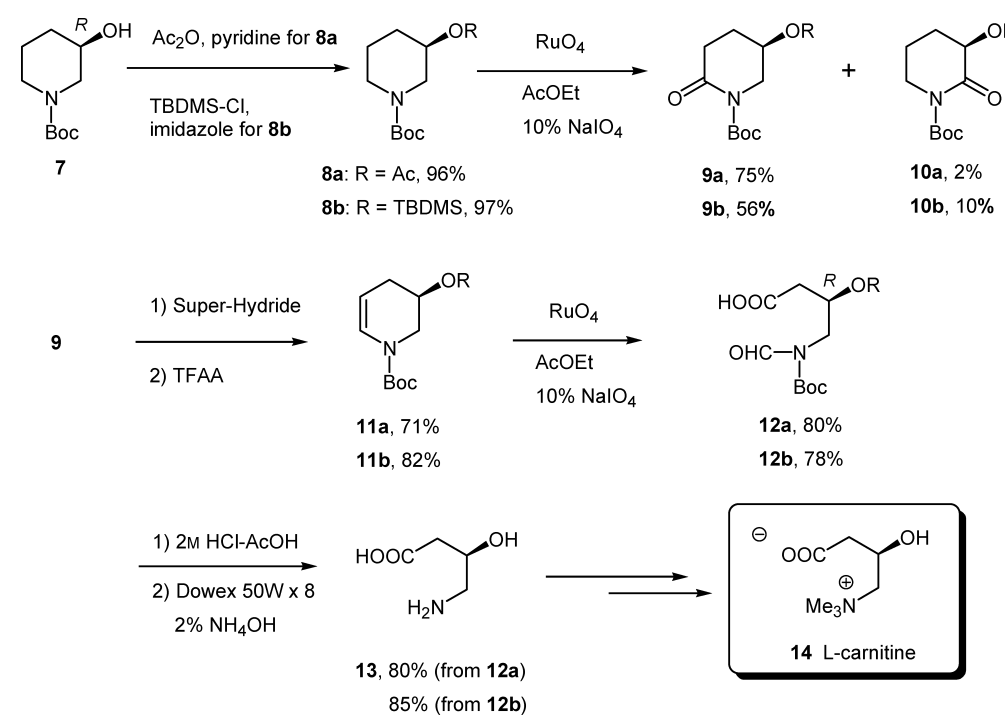

Chart 2 
complished.

\section{Experimental}

General Procedures Melting points were measured on a Yanagimoto micro melting point hot stage apparatus and are uncorrected. Optical rotations were measured on a JASCO DIP-370 digital polarimeter. IR spectra were determined with a Horiba FT-720 spectrometer. Mass spectra (MS) and HR-MS were recorded on a JEOL JMS-DX300 instrument. NMR spectra were determined with a JEOL EX-90A $(90 \mathrm{MHz})$ or a JEOL JNM-GSX 400 $(400 \mathrm{MHz})$ spectrometer in $\mathrm{CDCl}_{3}$ or $\mathrm{D}_{2} \mathrm{O}$ using tetramethylsilane or 1,4dioxane ( $\mathrm{H}, \delta 3.70 \mathrm{ppm}, \mathrm{C}, \delta 67.40 \mathrm{ppm})$ as internal standard and $J$ values are given in Hz. Microanalyses were performed in the Microanalytical Laboratory in this Faculty.

Starting Cyclic Ene-carbamates Unsubstituted $N$-protected cyclic enecarbamates $\left(\mathbf{1} \mathbf{a}-\mathbf{3 a},{ }^{19)} \mathbf{4 a}^{20-22)}\right)$ were prepared by the reported method. New compounds, $\left(\mathbf{5 a},{ }^{23)} \mathbf{6 \mathbf { a } ^ { 1 9 }}\right)$ were prepared from the corresponding lactams according to the procedure.

1-tert-Butyl 2-Methyl (2S)-1,2,3,4-Tetrahydropyridine-1,2-dicarboxylate (5a): Colorless oil; ${ }^{1} \mathrm{H}-\mathrm{NMR}\left(\mathrm{CDCl}_{3}\right) \delta: 1.46$ and $1.50(9 \mathrm{H}$, each s, intensity ratio $4: 5, t-\mathrm{Bu}), 1.83-2.04$ and $2.24-2.39(3 \mathrm{H}$ and $1 \mathrm{H}$, each $\mathrm{m}, 3-$ and $4-$ $\left.\mathrm{H}_{2}\right), 3.73$ and $3.74(3 \mathrm{H}$, each $\mathrm{s}$, intensity ratio $3: 2, \mathrm{OMe}), 4.72-4.76$ and $4.79-4.84(1 \mathrm{H}$, each $\mathrm{m}$, intensity ratio $2: 3,2-\mathrm{H}), 4.88-4.95(1 \mathrm{H}, \mathrm{m}, 5-\mathrm{H})$, 6.78 and $6.91(1 \mathrm{H}$, each d, intensity ratio $3: 2, J=8.5,8.4 \mathrm{~Hz}, 6-\mathrm{H}) ;{ }^{13} \mathrm{C}-$ NMR $\left(\mathrm{CDCl}_{3}\right) \delta: 18.32$ and 18.58 (each t), 23.50 and 23.67 (each t), 28.17 and 28.26 (each q), 52.24 and 52.33 (each q), 53.16 and 54.39 (each d), 81.17 and 81.36 (each s), 104.20 and 104.64 (each d), 124.45 and 124.91 (each d), 152.28 and 152.44 (each s), 171.58 and 172.00 (each s); IR (KBr) $\mathrm{cm}^{-1}:$ 1753, $1709(\mathrm{C}=\mathrm{O})$; MS (EI) $m / z: 241\left(\mathrm{M}^{+}\right)$; HR-MS $m / z: 241.1317$ (Calcd for $\left.\mathrm{C}_{12} \mathrm{H}_{19} \mathrm{NO}_{4}: 241.1314\right) ;[\alpha]_{\mathrm{D}}^{21}-28.0\left(c=1.41, \mathrm{CHCl}_{3}\right)$.

1-tert-Butyl 2-(Dimethoxyphosphoryl)-2,3-dihydropyrrole-1-carboxylate (6a): Colorless oil; ${ }^{1} \mathrm{H}-\mathrm{NMR}\left(\mathrm{CDCl}_{3}\right) \delta: 1.50(9 \mathrm{H}, \mathrm{s}, t-\mathrm{Bu}), 2.85-3.13(2 \mathrm{H}$, $\left.\mathrm{m}, 3-\mathrm{H}_{2}\right), 3.78\left(3 \mathrm{H}, \mathrm{d},{ }^{3} J_{\mathrm{HP}}=11.0 \mathrm{~Hz}, \mathrm{OMe}\right), 3.80\left(3 \mathrm{H}, \mathrm{d},{ }^{3} J_{\mathrm{HP}}=11.0 \mathrm{~Hz}\right.$, $\mathrm{OMe}), 4.43-4.61(1 \mathrm{H}, \mathrm{m}, 2-\mathrm{H}), 4.98-5.14(1 \mathrm{H}, \mathrm{br}, 4-\mathrm{H}), 6.34-6.64(1 \mathrm{H}$ br, 5-H); ${ }^{13} \mathrm{C}-\mathrm{NMR}\left(\mathrm{CDCl}_{3}\right) \delta: 28.26$ (q), 31.74 (brt), 51.61 (brd), 53.01 and $53.45\left(\right.$ each $\left.\mathrm{d}_{\mathrm{p}} \mathrm{q},{ }^{2} J_{\mathrm{CP}}=6.7 \mathrm{~Hz}\right), 81.09(\mathrm{~s}), 107.51\left(\mathrm{~d}_{\mathrm{P}} \mathrm{d},{ }^{3} J_{\mathrm{CP}}=1.9 \mathrm{~Hz}\right)$, 130.13 (d), 151.92 (s); IR (KBr) cm $\mathrm{cm}^{-1}: 1703(\mathrm{C}=\mathrm{O})$; MS (EI) $\mathrm{m} / z: 277$ $\left(\mathrm{M}^{+}\right)$; HR-MS $m / z$ : 277.1078 (Calcd for $\mathrm{C}_{11} \mathrm{H}_{20} \mathrm{NO}_{5} \mathrm{P}: 277.1079$ ).

General Procedure for $\mathrm{RuO}_{4}$ Oxidation of $\mathrm{N}$-Protected Cyclic Enecarbamates A solution of a substrate $(12 \mathrm{mmol})$ to be oxidized in ethyl acetate $(120 \mathrm{ml})$ was added to a mixture of $\mathrm{RuO}_{2} \cdot x$ hydrate $(80 \mathrm{mg})$ and $10 \%$ $\mathrm{NaIO}_{4}$ aqueous solution $(320 \mathrm{ml})$. The reaction mixture was vigorously stirred in a sealed flask at $0{ }^{\circ} \mathrm{C}$ until disappearance of the starting materials. The layers were separated, and the aqueous layer was extracted with ethyl acetate $(100 \mathrm{ml} \times 3)$. The combined organic layer was stirred with isopropanol $(2 \mathrm{ml})$ to decompose the $\mathrm{RuO}_{4}$ for $2-3 \mathrm{~h}$ and filtered off. The filtrate was washed with brine $(40 \mathrm{ml} \times 2)$ and dried over anhydrous $\mathrm{Na}_{2} \mathrm{SO}_{4}$. The organic solvent was evaporated in vacuo to leave a residue, which was purified by silica gel chromatography using ethyl acetate-hexane $(3: 1)$ as an eluent to give the $\omega$-( $N$-formylamino)carboxylic acid.

3-( $N$-tert-Butoxycarbonyl- $N$-formylamino)propionic Acid (1b): Colorless oil; ${ }^{1} \mathrm{H}-\mathrm{NMR}\left(\mathrm{CDCl}_{3}\right) \delta: 1.55(9 \mathrm{H}, \mathrm{s}, t-\mathrm{Bu}), 2.61\left(2 \mathrm{H}, \mathrm{t}, J=7.4 \mathrm{~Hz}, 2-\mathrm{H}_{2}\right)$, $3.91\left(2 \mathrm{H}, \mathrm{t}, J=7.4 \mathrm{~Hz}, 3-\mathrm{H}_{2}\right), 8.50-9.34(2 \mathrm{H}, \mathrm{br}, \mathrm{CHO}$ and $\mathrm{COOH}) ;{ }^{13} \mathrm{C}-$ $\operatorname{NMR}\left(\mathrm{CDCl}_{3}\right) \delta: 27.98(\mathrm{q}), 32.65(\mathrm{t}), 36.16(\mathrm{t}), 84.60(\mathrm{~s}), 152.01(\mathrm{~s}), 162.99$ (d), 176.32 (s); IR (KBr) cm ${ }^{-1}: 3317(\mathrm{OH}), 1738,1712,1693(\mathrm{C}=\mathrm{O})$; MS (FAB) $m / z: 218\left(\mathrm{MH}^{+}\right)$; HR-MS (FAB) $m / z: 218.1029\left(\right.$ Calcd for $\mathrm{C}_{9} \mathrm{H}_{16} \mathrm{NO}_{5}$ : 218.1028).

4-(N-tert-Butoxycarbonyl- $N$-formylamino)butyric Acid (2b): Colorless oil; ${ }^{1} \mathrm{H}-\mathrm{NMR}\left(\mathrm{CDCl}_{3}\right) \delta: 1.55(9 \mathrm{H}, \mathrm{s}, t$-Bu $), 1.82-1.96\left(2 \mathrm{H}, \mathrm{m}, 3-\mathrm{H}_{2}\right), 2.38$ $\left(2 \mathrm{H}, \mathrm{t}, J=7.4 \mathrm{~Hz}, 2-\mathrm{H}_{2}\right), 3.67\left(2 \mathrm{H}, J=7.1 \mathrm{~Hz}, 4-\mathrm{H}_{2}\right), 8.42-9.49(2 \mathrm{H}, \mathrm{br}$, $\mathrm{CHO}$ and $\mathrm{COOH}) ;{ }^{13} \mathrm{C}-\mathrm{NMR}\left(\mathrm{CDCl}_{3}\right) \delta: 23.22(\mathrm{t}), 27.98(\mathrm{q}), 31.18(\mathrm{t})$, 39.73 (t), 84.31 (s), 152.40 (s), 163.33 (d), 178.40 (s); IR (KBr) cm ${ }^{-1}: 3201$ $(\mathrm{OH}), 1738,1705,1693(\mathrm{C}=\mathrm{O})$; MS (FAB) $m / z: 232\left(\mathrm{MH}^{+}\right)$; HR-MS (FAB) $m / z: 232.1088$ (Calcd for $\mathrm{C}_{10} \mathrm{H}_{18} \mathrm{NO}_{5}: 232.1185$ ).

5-( $N$-tert-Butoxycarbonyl- $N$-formylamino)pentanoic Acid (3b): Colorless oil; ${ }^{1} \mathrm{H}-\mathrm{NMR}\left(\mathrm{CDCl}_{3}\right) \delta: 1.55(9 \mathrm{H}, \mathrm{s}, t-\mathrm{Bu}), 1.57-1.69(4 \mathrm{H}, \mathrm{m}, 3-$ and $4-$ $\left.\mathrm{H}_{2}\right), 2.39\left(2 \mathrm{H}, \mathrm{t}, J=7.1 \mathrm{~Hz}, 2-\mathrm{H}_{2}\right), 3.61\left(2 \mathrm{H}, J=6.9 \mathrm{~Hz}, 5-\mathrm{H}_{2}\right), 8.75-11.30$ $(2 \mathrm{H}, \mathrm{br}, \mathrm{CHO}$ and $\mathrm{COOH}) ;{ }^{13} \mathrm{C}-\mathrm{NMR}\left(\mathrm{CDCl}_{3}\right) \delta: 21.80(\mathrm{t}), 27.65(\mathrm{t}), 28.02$ (q), $33.50(\mathrm{t}), 40.04(\mathrm{t}), 84.11(\mathrm{~s}), 152.48(\mathrm{~s}), 163.25$ (d), $179.15(\mathrm{~s}) ;$ IR $(\mathrm{KBr}) \mathrm{cm}^{-1}: 3224(\mathrm{OH}), 1736,1705,1691(\mathrm{C}=\mathrm{O})$; MS (FAB) $\mathrm{m} / \mathrm{z}: 246$ $\left(\mathrm{MH}^{+}\right.$); HR-MS (FAB) $m / z: 246.1344$ (Calcd for $\mathrm{C}_{11} \mathrm{H}_{20} \mathrm{NO}_{5}: 246.1341$ ).

(2S)-2-(N-tert-Butoxycarbonyl- $N$-formylamino)succinic Acid 1-Methyl Ester (4b): Colorless oil; ${ }^{1} \mathrm{H}-\mathrm{NMR}\left(\mathrm{CDCl}_{3}\right) \delta: 1.54(9 \mathrm{H}, \mathrm{s}, t-\mathrm{Bu}), 2.75$ and 3.31 (each $1 \mathrm{H}$, dd, $J=16.9,6.6$ and $\left.16.9,7.3 \mathrm{~Hz}, 3-\mathrm{H}_{2}\right), 3.74(3 \mathrm{H}, \mathrm{s}$, COOMe), $5.52(1 \mathrm{H}, \mathrm{dd}, J=7.3,6.6 \mathrm{~Hz}, 2-\mathrm{H}), 7.28(1 \mathrm{H}, \mathrm{br}$ s, CHO $), 9.16$
$(1 \mathrm{H}, \mathrm{s}, \mathrm{COOH}) ;{ }^{13} \mathrm{C}-\mathrm{NMR}\left(\mathrm{CDCl}_{3}\right) \delta: 27.93(\mathrm{q}), 34.53(\mathrm{t}), 49.26(\mathrm{~d}), 52.86$ (q), 85.55 (s), 151.30 (s), 162.23 (d), 169.20 (s), 175.27 (s); IR (KBr) cm ${ }^{-1}$ : $3452(\mathrm{OH}), 1747,1712,1699(\mathrm{C}=\mathrm{O})$; MS (FAB) $m / z: 276\left(\mathrm{MH}^{+}\right)$; HR-MS (FAB) $m / z: 276.1082$ (Calcd for $\mathrm{C}_{11} \mathrm{H}_{18} \mathrm{NO}_{7}: 276.1083$ ); $[\alpha]_{\mathrm{D}}^{21}-75.9$ $\left(c=1.0, \mathrm{CHCl}_{3}\right)$.

(2S)-2-(N-tert-Butoxycarbonyl- $N$-formylamino)pentanedioic Acid 1Methyl Ester (5b): Colorless oil; ${ }^{1} \mathrm{H}-\mathrm{NMR}\left(\mathrm{CDCl}_{3}\right) \delta$ : $1.52(9 \mathrm{H}, \mathrm{s}, t$-Bu), $2.14-2.25$ and $2.33-2.55\left(1 \mathrm{H}\right.$ and $3 \mathrm{H}$, each $\mathrm{m}, 3-$ and $\left.4-\mathrm{H}_{2}\right), 3.73(3 \mathrm{H}, \mathrm{s}$, OMe), $5.05(1 \mathrm{H}, \mathrm{dd}, J=9.7,5.0 \mathrm{~Hz}, 2-\mathrm{H}), 7.45-8.98(1 \mathrm{H}, \mathrm{br}, \mathrm{COOH}), 9.22$ $(1 \mathrm{H}, \mathrm{s}, \mathrm{CHO}) ;{ }^{13} \mathrm{C}-\mathrm{NMR}\left(\mathrm{CDCl}_{3}\right) \delta: 24.09$ (t), 27.91 (q), 30.46 (t), 52.14 (d), 52.51 (q), 85.30 (s), 151.55 (s), 162.80 (d), 169.77 (s), 178.09 (s); IR $(\mathrm{KBr}) \mathrm{cm}^{-1}: 3384(\mathrm{OH}), 1743,1711(\mathrm{C}=\mathrm{O})$; MS (FAB) $m / z: 290\left(\mathrm{MH}^{+}\right)$. HR-MS (FAB) $\mathrm{m} / \mathrm{z}: 290.1242$ (Calcd for $\mathrm{C}_{12} \mathrm{H}_{20} \mathrm{NO}_{7}: 290.1240$ ); $[\alpha]_{\mathrm{D}}^{21}$ $-30.5(c=6.4, \mathrm{MeOH})$.

3-(N-tert-Butoxycarbonyl- $N$-formylamino)-3-(dimethoxyphosphoryl)propionic Acid (6b): Colorless oil; ${ }^{1} \mathrm{H}-\mathrm{NMR}\left(\mathrm{CDCl}_{3}\right) \delta: 1.56(9 \mathrm{H}, \mathrm{s}, t-\mathrm{Bu})$, $2.79-3.01$ and $3.06-3.28$ (each $1 \mathrm{H}, \mathrm{m}, 2-\mathrm{H}_{2}$ ), 3.75 and 3.82 (each $3 \mathrm{H}, \mathrm{d}$, $\left.{ }^{3} J_{\mathrm{HP}}=11.0 \mathrm{~Hz}, \mathrm{OMe} \times 2\right), 5.06-5.45(1 \mathrm{H}, \mathrm{m}, 3-\mathrm{H}), 9.13(1 \mathrm{H}, \mathrm{s}, \mathrm{CHO})$, 9.16-9.45 (1H, br, $\mathrm{COOH}) ;{ }^{13} \mathrm{C}-\mathrm{NMR}\left(\mathrm{CDCl}_{3}\right) \delta: 27.88(\mathrm{q}), 32.53(\mathrm{t})$, $43.11\left(\mathrm{~d}_{\mathrm{P}} \mathrm{d},{ }^{1} J_{\mathrm{CP}}=149.5 \mathrm{~Hz}\right), 53.09$ and $55.21\left(\right.$ each d $\mathrm{d}_{\mathrm{P}}$, $\left.{ }^{2} J_{\mathrm{CP}}=6.7 \mathrm{~Hz}\right), 85.45$ (s), $151.52(\mathrm{~s}), 162.67(\mathrm{~d}), 172.77\left(\mathrm{~d}_{\mathrm{p}},{ }^{3} J_{\mathrm{CP}}=16.3 \mathrm{~Hz}\right) ; \mathrm{IR}(\mathrm{KBr}) \mathrm{cm}^{-1}: 3423$ (OH), $1699(\mathrm{C}=\mathrm{O})$; MS m/z: $326\left(\mathrm{MH}^{+}\right)$; HR-MS (FAB) $m / z: 326.1008$ (Calcd for $\mathrm{C}_{11} \mathrm{H}_{21} \mathrm{NO}_{8} \mathrm{P}: 326.1005$ ).

tert-Butyl (3R)-3-Hydroxypiperidine-1-carboxylate (7) This compound was synthesized by the reported method. ${ }^{27)}$ Colorless oil; $[\alpha]_{\mathrm{D}}^{20}-10.8$ $\left(c=1.11, \mathrm{CHCl}_{3}\right) ;$ lit. $^{27)}[\alpha]_{\mathrm{D}}^{20}-9.6\left(c=1, \mathrm{CHCl}_{3}\right)$.

tert-Butyl (3R)-3-Acetoxypiperidine-1-carboxylate (8a) $\quad \mathrm{Ac}_{2} \mathrm{O}(3.67 \mathrm{~g}$, $36 \mathrm{mmol})$ was slowly added to a solution of $7(6.04 \mathrm{~g}, 30 \mathrm{mmol})$ in pyridine $(50 \mathrm{ml})$ with stirring at $0{ }^{\circ} \mathrm{C}$. The reaction mixture was allowed to warm to room temperature, stirred for $24 \mathrm{~h}$. The mixture was diluted with benzene $(300 \mathrm{ml})$ and saturated aqueous $\mathrm{NaHCO}_{3}(150 \mathrm{ml})$. The organic layer was washed with water $(50 \mathrm{ml} \times 3)$, dried over anhydrous $\mathrm{Na}_{2} \mathrm{SO}_{4}$, and evaporated to furnish the crude product which was purified by chromatography (silica gel, AcOEt-hexane, 1:5). $7.04 \mathrm{~g}(96 \%)$; colorless oil; $[\alpha]_{\mathrm{D}}^{20}+22.6(c=2.46$, $\left.\mathrm{CHCl}_{3}\right) ;$ lit. $^{27)}[\alpha]_{\mathrm{D}}^{20}+21.2\left(c=1.1, \mathrm{CHCl}_{3}\right)$. This compound was identical with the authentic sample prepared in the previous paper. ${ }^{27)}$

tert-Butyl (3R)-3-(tert-Butyldimethylsilyloxy)piperidine-1-carboxylate (8b) tert-Butyldimethylsilyl chloride $(5.65 \mathrm{~g}, 37.5 \mathrm{mmol})$ was slowly added to a solution of $7(6.04 \mathrm{~g}, 30 \mathrm{mmol})$ and imidazole $(8.17 \mathrm{~g}, 120 \mathrm{mmol})$ in dry DMF $(50 \mathrm{ml})$ with stirring at $0{ }^{\circ} \mathrm{C}$. The reaction mixture was allowed to warm to room temperature, stirred for $24 \mathrm{~h}$, and poured into ice-water $(100 \mathrm{ml})$. The aqueous mixture was extracted with AcOEt $(100 \mathrm{ml} \times 3)$, organic layer was washed with brine $(50 \mathrm{ml} \times 3)$, dried over anhydrous $\mathrm{Na}_{2} \mathrm{SO}_{4}$, and evaporated to furnish the crude product which was purified by chromatography (silica gel, AcOEt-hexane, 1:5). $9.22 \mathrm{~g}$ (97\%); colorless oil; ${ }^{1} \mathrm{H}-\mathrm{NMR}\left(\mathrm{CDCl}_{3}\right) \delta: 0.07$ and $0.08\left(\right.$ each $\left.3 \mathrm{H}, \mathrm{s}, \mathrm{SiMe}_{2}\right), 0.89(9 \mathrm{H}, \mathrm{s}, \mathrm{Sit}-$ $\mathrm{Bu}), 1.45(9 \mathrm{H}, \mathrm{s}, \mathrm{COO} t-\mathrm{Bu}), 1.35-1.52\left(2 \mathrm{H}, \mathrm{m}, 5-\mathrm{H}_{2}\right), 1.66-1.76$ and $1.80-1.90\left(\right.$ each $\left.1 \mathrm{H}, \mathrm{m}, 4-\mathrm{H}_{2}\right), 2.70-2.97\left(2 \mathrm{H}, \mathrm{m}, 6-\mathrm{H}_{2}\right), 3.53-3.94(3 \mathrm{H}$, $\mathrm{m}, 2-\mathrm{H}_{2}$ and $\left.3-\mathrm{H}\right) ;{ }^{13} \mathrm{C}-\mathrm{NMR}\left(\mathrm{CDCl}_{3}\right) \delta:-4.74(\mathrm{q}), 18.13(\mathrm{~s}), 23.04(\mathrm{t})$ $25.82(\mathrm{q}), 28.44(\mathrm{q}), 33.90(\mathrm{t}), 43.36$ and $44.10(\mathrm{t}), 50.51$ and $51.54(\mathrm{t})$, 67.14 (d), 79.30 (s), 154.78 (s); IR (KBr) cm cm $^{-1} 1699(\mathrm{C}=\mathrm{O})$; MS (FAB) $m / z$ : $316\left(\mathrm{MH}^{+}\right)$; HR-MS (FAB) $m / z: 316.2313$ (Calcd for $\mathrm{C}_{16} \mathrm{H}_{34} \mathrm{NO}_{3} \mathrm{Si}$ : 316.2308); $[\alpha]_{\mathrm{D}}^{20}-14.1\left(c=1.31, \mathrm{CHCl}_{3}\right)$.

$\mathrm{RuO}_{4}$ Oxidation of $\mathrm{N}$-Boc-piperidines (8) A solution of piperidine (8, $24 \mathrm{mmol})$ was oxidized in ethyl acetate $(80 \mathrm{ml})$ was added to a mixture of $\mathrm{RuO}_{2} \cdot x \mathrm{H}_{2} \mathrm{O}(200 \mathrm{mg})$ and $10 \%$ aqueous $\mathrm{NaIO}_{4}$ solution $(240 \mathrm{ml})$. The reaction mixture was stirred in a sealed flask at $20^{\circ} \mathrm{C}$ until disappearance of the starting material, and worked up as described above for the general procedure for $\mathrm{RuO}_{4}$ oxidation of $N$-protected cyclic ene-carbamates, and the 3substituted 2-oxopiperidines (10, the first fractions) and 5-substituted 2-oxo derivatives $(\mathbf{9}$, the second fractions) could be separated by silica gel chromatography.

tert-Butyl (5R)-5-Acetoxy-2-oxopiperidine-1-carboxylate $\quad(9 \mathrm{a}): 4.63 \mathrm{~g}$ (75\%); colorless oil; ${ }^{1} \mathrm{H}-\mathrm{NMR}\left(\mathrm{CDCl}_{3}\right) \delta: 1.53(9 \mathrm{H}, \mathrm{s}, t$-Bu), $1.95-2.08$ and $2.11-2.18$ (each $\left.1 \mathrm{H}, \mathrm{m}, 4-\mathrm{H}_{2}\right), 2.08(3 \mathrm{H}, \mathrm{s}, \mathrm{OAc}), 2.47-2.55$ and 2.61-2.69 (each $1 \mathrm{H}, \mathrm{m}, 3-\mathrm{H}_{2}$ ), 3.72 and 3.93 (each $1 \mathrm{H}$, dd and ddd, $J=14.0,3.8$ and $\left.14.0,4.0,1.6 \mathrm{~Hz}, 6-\mathrm{H}_{2}\right), 5.18-5.24(1 \mathrm{H}, \mathrm{m}, 5-\mathrm{H}) ;{ }^{13} \mathrm{C}-$ $\operatorname{NMR}\left(\mathrm{CDCl}_{3}\right) \delta: 21.07(\mathrm{q}), 25.26(\mathrm{t}), 27.98(\mathrm{q}), 31.03(\mathrm{t}), 49.02(\mathrm{t}), 66.20$ (d), 83.35 (s), 152.01 (s), 170.19 (s), 170.20 (s); IR (KBr) cm ${ }^{-1}: 1774,1738$, $1722(\mathrm{C}=\mathrm{O})$; MS (FAB) $m / z: 258\left(\mathrm{MH}^{+}\right)$. HR-MS (FAB) $m / z: 258.1339$ (Calcd for $\left.\mathrm{C}_{12} \mathrm{H}_{20} \mathrm{NO}_{5}: 258.1342\right) ;[\alpha]_{\mathrm{D}}^{25}+13.0\left(c=1.5, \mathrm{CHCl}_{3}\right)$.

tert-Butyl (3R)-3-Acetoxy-2-oxopiperidine-1-carboxylate (10a): $124 \mathrm{mg}$ (2\%), colorless oil; ${ }^{1} \mathrm{H}-\mathrm{NMR}\left(\mathrm{CDCl}_{3}\right) \delta: 1.52(9 \mathrm{H}, \mathrm{s}, t-\mathrm{Bu}), 1.86-2.04$ and $2.20-2.29\left(3 \mathrm{H}\right.$ and $1 \mathrm{H}$, each $\mathrm{m}, 4-$ and $\left.5-\mathrm{H}_{2}\right), 2.16(3 \mathrm{H}, \mathrm{s}, \mathrm{OAc}), 3.60-$ 
3.67 and 3.76-3.84 (each $\left.1 \mathrm{H}, \mathrm{m}, 6-\mathrm{H}_{2}\right), 5.31(1 \mathrm{H}, \mathrm{dd}, J=10.8,7.3 \mathrm{~Hz}, 3-$ $\mathrm{H}) ;{ }^{13} \mathrm{C}-\mathrm{NMR}\left(\mathrm{CDCl}_{3}\right) \delta: 20.27(\mathrm{t}), 20.81(\mathrm{q}), 26.23(\mathrm{t}), 27.97(\mathrm{q}), 44.86(\mathrm{t})$, 69.99 (d), 83.40 (s), 152.43 (s), 168.00 (s), 169.96 (s); IR (KBr) cm ${ }^{-1}: 1776$, 1738, $1722(\mathrm{C}=\mathrm{O})$; MS (EI) $m / z: 257\left(\mathrm{M}^{+}\right)$. HR-MS (EI) $m / z: 257.1258$ $\left(\right.$ Calcd for $\left.\mathrm{C}_{12} \mathrm{H}_{19} \mathrm{NO}_{5}: 257.1263\right) ;[\alpha]_{\mathrm{D}}^{25}+51.4\left(c=1.1, \mathrm{CHCl}_{3}\right)$.

tert-Butyl (5R)-5-(tert-Butyldimethylsilyloxy)-2-oxopiperidine-1-carboxylate (9b): $4.42 \mathrm{~g}(56 \%)$; colorless oil; ${ }^{1} \mathrm{H}-\mathrm{NMR}\left(\mathrm{CDCl}_{3}\right) \delta: 0.09(6 \mathrm{H}, \mathrm{s}$, $\left.\mathrm{SiMe}_{2}\right), 0.89(9 \mathrm{H}, \mathrm{s}, \mathrm{Sit}-\mathrm{Bu}), 1.52(9 \mathrm{H}, \mathrm{s}, \mathrm{COO} t-\mathrm{Bu}), 1.79-1.88$ and $1.92-$ $2.01\left(\right.$ each $\left.1 \mathrm{H}, \mathrm{m}, 4-\mathrm{H}_{2}\right), 2.39-2.47$ and $2.67-2.76$ (each $1 \mathrm{H}, \mathrm{m}, 3-\mathrm{H}_{2}$ ), $3.60-3.72\left(2 \mathrm{H}, \mathrm{m}, 6-\mathrm{H}_{2}\right), 4.13-4.19(1 \mathrm{H}, \mathrm{m}, 5-\mathrm{H}) ;{ }^{13} \mathrm{C}-\mathrm{NMR}\left(\mathrm{CDCl}_{3}\right) \delta$ : $-4.88(\mathrm{q}), 17.99(\mathrm{~s}), 25.68(\mathrm{q}), 28.00(\mathrm{q}), 29.00(\mathrm{t}), 31.06(\mathrm{t}), 52.42(\mathrm{t})$, 64.44 (d), 82.83 (s), 152.49 (s), 170.90 (s); IR (KBr) $\mathrm{cm}^{-1}: 1774,1716$ $(\mathrm{C}=\mathrm{O})$; MS (FAB) $\mathrm{m} / \mathrm{z}: 330\left(\mathrm{MH}^{+}\right)$. HR-MS (FAB) $\mathrm{m} / z: 330.2103$ (Calcd for $\left.\mathrm{C}_{16} \mathrm{H}_{32} \mathrm{NO}_{4} \mathrm{Si}: 330.2101\right) ;[\alpha]_{\mathrm{D}}^{24}-8.1\left(c=0.95, \mathrm{CHCl}_{3}\right)$.

tert-Butyl (3R)-3-(tert-Butyldimethylsilyloxy)-2-oxopiperidine-1-carboxylate (10b): $0.79 \mathrm{~g}(10 \%)$; colorless oil; ${ }^{1} \mathrm{H}-\mathrm{NMR}\left(\mathrm{CDCl}_{3}\right) \delta: 0.15$ and 0.17 $\left(6 \mathrm{H}\right.$, each s, intensity ratio $\left.9: 11, \mathrm{SiMe}_{2}\right), 0.90$ and $0.91(9 \mathrm{H}$, each $\mathrm{s}$, intensity ratio $4: 5$, Sit-Bu), 1.51 and $1.56(9 \mathrm{H}$, each $\mathrm{s}$, intensity ratio $4: 5, \mathrm{COO} t$ $\mathrm{Bu}), 1.71-2.13\left(4 \mathrm{H}, \mathrm{m}, 4-\right.$ and $\left.5-\mathrm{H}_{2}\right), 6-\mathrm{H}_{2}$ [2.57-2.66 and $2.84-2.93$ $(1 \mathrm{H}$, each $\mathrm{m}$, intensity ratio $2: 3), 3.58-3.72(1 \mathrm{H}, \mathrm{m})], 4.17$ and $4.33(1 \mathrm{H}$, each dd, intensity ratio $2: 3, J=8.2,5.6$ and $7.4,4.6 \mathrm{~Hz}, 3-\mathrm{H}) ;{ }^{13} \mathrm{C}-\mathrm{NMR}$ $\left(\mathrm{CDCl}_{3}\right) \delta:-5.56,-5.41,-4.77$ and -4.59 (each $\left.\mathrm{q}\right), 18.22$ and 18.32 (each s), 19.91 and 26.56 (each t), 25.63 and 25.77 (each q), 27.46 and 28.00 (each q), 28.70 and 30.46 (each t), 45.54 (t), 68.74 and 71.54 (each d), 82.72 and 86.31 (each s), 148.61 and 152.74 (each s), 169.63 and 171.98 (each s); IR (KBr) cm ${ }^{-1}: 1778,1707(\mathrm{C}=\mathrm{O})$; MS (FAB) $\mathrm{m} / z: 330\left(\mathrm{MH}^{+}\right)$. HR-MS (FAB) $m / z: 330.2102$ (Calcd for $\mathrm{C}_{16} \mathrm{H}_{32} \mathrm{NO}_{4} \mathrm{Si}: 330.2101$ ); $[\alpha]_{\mathrm{D}}^{24}+16.2$ $\left(c=1.02, \mathrm{CHCl}_{3}\right)$

tert-Butyl (3R)-3-Acetoxy-1,2,3,4-tetrahydropyridine-1-carboxylate (11a) To a solution of the lactam $(9,2.57 \mathrm{~g}, 10 \mathrm{mmol})$ in toluene $(20 \mathrm{ml})$ at $-70^{\circ} \mathrm{C}$ Super-Hydride $(1.0 \mathrm{M}$ in THF, $10.6 \mathrm{ml})$ was added dropwise. After stirring at $-70^{\circ} \mathrm{C}$ for $30 \mathrm{~min}$, DIPEA $(10 \mathrm{ml}, 57 \mathrm{mmol})$, dimethylaminopyridine $(24 \mathrm{mg})$ and TFAA $(1.7 \mathrm{ml}, 12 \mathrm{mmol})$ were added. The mixture was warmed to room temperature and stirred for $2 \mathrm{~h}$, and water $(20 \mathrm{ml})$ was added to the mixture. Organic layer was separated and washed with brine $(30 \mathrm{ml} \times 3)$, dried over anhydrous $\mathrm{Na}_{2} \mathrm{SO}_{4}$, and evaporated to furnish the crude product which was purified by chromatography (silica gel, AcOEt-hexane, $1: 5)$. $1.71 \mathrm{~g}(71 \%)$; colorless oil; ${ }^{1} \mathrm{H}-\mathrm{NMR}\left(\mathrm{CDCl}_{3}\right) \delta: 1.47$ and $1.49(9 \mathrm{H}$, each $\mathrm{s}$, intensity ratio $4: 6, t-\mathrm{Bu}), 2.06(3 \mathrm{H}, \mathrm{s}, \mathrm{OAc}), 2.12$ 2.18 and $2.36-2.43$ (each $\left.1 \mathrm{H}, \mathrm{m}, 4-\mathrm{H}_{2}\right), 3.56-3.74\left(2 \mathrm{H}, \mathrm{m}, 2-\mathrm{H}_{2}\right), 4.69-$ $4.87(1 \mathrm{H}, \mathrm{m}, 3-\mathrm{H}), 5.08-5.21(1 \mathrm{H}, \mathrm{br}, 5-\mathrm{H}), 6.76$ and $6.88(1 \mathrm{H}$, each $\mathrm{d}$, intensity ratio $3: 2, J=8.2$ and $7.1 \mathrm{~Hz}, 6-\mathrm{H}) ;{ }^{13} \mathrm{C}-\mathrm{NMR}\left(\mathrm{CDCl}_{3}\right) \delta: 21.17$ (q), 27.22 and 27.35 (each t), $28.28(\mathrm{q}), 44.15$ and $45.14(\mathrm{t}), 65.76$ and 65.92 (each d), 81.08 (s), 101.27 and 101.73 (each d), 125.42 and 125.77 (each d), 152.35 and 152.67 (each s), $170.40(\mathrm{~s}) ; \mathrm{IR}(\mathrm{KBr}) \mathrm{cm}^{-1}$ : 1739, $1705(\mathrm{C}=\mathrm{O})$; MS (EI) $m / z: 241\left(\mathrm{M}^{+}\right)$. HR-MS (EI) $m / z$ : $241.1312\left(\right.$ Calcd for $\mathrm{C}_{12} \mathrm{H}_{19} \mathrm{NO}_{4}$ : 241.1314); $[\alpha]_{\mathrm{D}}^{23}+18.4\left(c=1.06, \mathrm{CHCl}_{3}\right)$.

tert-Butyl (3R)-3-(tert-Butyldimethylsilyloxy)-1,2,3,4-tetrahydropyridine-1-carboxylate (11b) This compound was prepared from $9 \mathrm{~b}$ in a similar manner to that described for 11a. $2.57 \mathrm{~g}(82 \%)$; colorless oil; ${ }^{1} \mathrm{H}-\mathrm{NMR}$ $\left(\mathrm{CDCl}_{3}\right) \delta: 0.08$ and 0.09 (each $\left.3 \mathrm{H}, \mathrm{s}, \mathrm{SiMe}_{2}\right), 0.88(9 \mathrm{H}, \mathrm{s}, \mathrm{Sit}-\mathrm{Bu}), 1.49$ $(9 \mathrm{H}, \mathrm{s}, \mathrm{COO} t-\mathrm{Bu}), 1.96-2.06$ and $2.19-2.29$ (each $\left.1 \mathrm{H}, \mathrm{m}, 4-\mathrm{H}_{2}\right), 2-\mathrm{H}_{2}$ [3.06-3.19 $(1 \mathrm{H}, \mathrm{m}), 3.73$ and $3.84(1 \mathrm{H}$, each d, intensity ratio $3: 7, J=11.7$ and $12.0 \mathrm{~Hz})] .3 .95-4.04(1 \mathrm{H}, \mathrm{m}, 3-\mathrm{H}), 4.66-4.88(1 \mathrm{H}, \mathrm{m}, 5-\mathrm{H}), 6.67$ and $6.81(1 \mathrm{H}$, each d, intensity ratio $7: 3, J=8.1$ and $7.8 \mathrm{~Hz}, 6-\mathrm{H}) ;{ }^{13} \mathrm{C}-\mathrm{NMR}$ $\left(\mathrm{CDCl}_{3}\right) \delta:-4.76$ and -4.68 (each q), 18.06 and 18.12 (each s), 25.78 (q), 28.32 (q), 31.71 and 31.85 (each t), 47.54 and 48.72 (each t), 64.83 (d), 80.56 and 80.69 (each s), 102.37 and 102.94 (each d), 125.08 and 125.40 (each d), 152.29 and 152.65 (each s); IR $\left(\mathrm{KBr} \mathrm{cm}^{-1}: 1705(\mathrm{C}=\mathrm{O})\right.$; MS (EI) $m / z: 313\left(\mathrm{M}^{+}\right)$; HR-MS (EI) $\mathrm{m} / z$ : 313.2070 (Calcd for $\mathrm{C}_{16} \mathrm{H}_{31} \mathrm{NO}_{3} \mathrm{Si}$ : $313.2073) ;[\alpha]_{\mathrm{D}}^{25}-16.5\left(c=1.18, \mathrm{CHCl}_{3}\right)$.

(3R)-3-Acetoxy-4-( $N$-tert-butoxycarbonyl- $N$-formylamino)butyric Acid (12a) The enamine (11, $24 \mathrm{mmol}$ ) was oxidized and worked up as described above for the general procedure for $\mathrm{RuO}_{4}$ oxidation of $\mathrm{N}$-protected cyclic ene-carbamates. $5.57 \mathrm{~g}(80 \%)$; colorless oil; ${ }^{1} \mathrm{H}-\mathrm{NMR}\left(\mathrm{CDCl}_{3}\right) \delta$ : $1.56(9 \mathrm{H}, \mathrm{s}, t-\mathrm{Bu}), 2.01(3 \mathrm{H}, \mathrm{s}, \mathrm{OAc}), 2.64\left(2 \mathrm{H}, \mathrm{d}, J=6.7 \mathrm{~Hz}, 2-\mathrm{H}_{2}\right), 3.81$ and 3.95 (each $1 \mathrm{H}$, dd, $J=14.2,3.8$ and $\left.14.2,6.9 \mathrm{~Hz}, 4-\mathrm{H}_{2}\right), 5.42-5.48(1 \mathrm{H}, \mathrm{m}$, $3-\mathrm{H}), 6.81-7.28(1 \mathrm{H}, \mathrm{br}, \mathrm{COOH}), 9.17(1 \mathrm{H}, \mathrm{s}, \mathrm{CHO}) ;{ }^{13} \mathrm{C}-\mathrm{NMR}\left(\mathrm{CDCl}_{3}\right) \delta$ : $20.87(\mathrm{q}), 27.95$ (q), $36.75(\mathrm{t}), 42.47(\mathrm{t}), 67.90$ (d), $84.87(\mathrm{~s}), 152.13(\mathrm{~s})$, 163.44 (d), 170.53 (s), 174.48 (s); IR (KBr) cm $\mathrm{cm}^{-1}: 3482(\mathrm{OH}), 1743,1711$, $1693(\mathrm{C}=\mathrm{O})$; MS (FAB) $m / z: 290\left(\mathrm{MH}^{+}\right)$. HR-MS (FAB) $\mathrm{m} / z: 290.1242$ (Calcd for $\left.\mathrm{C}_{12} \mathrm{H}_{20} \mathrm{NO}_{7}: 290.1240\right) ;[\alpha]_{\mathrm{D}}^{23}+10.0\left(c=1.99, \mathrm{CHCl}_{3}\right)$.

(3R)-4-( $N$-tert-Butoxycarbonyl- $N$-formylamino)-3-(tert-butyldimeth-
ylsilyloxy)Butyric Acid (12b) $6.77 \mathrm{~g}$, (78\%); colorless oil; ${ }^{1} \mathrm{H}-\mathrm{NMR}$ $\left(\mathrm{CDCl}_{3}\right) \delta$ : 0.06 and 0.08 (each $\left.3 \mathrm{H}, \mathrm{s}, \mathrm{SiMe}_{2}\right), 0.86(9 \mathrm{H}, \mathrm{s}, \mathrm{Sit}-\mathrm{Bu}), 1.55$ $(9 \mathrm{H}, \mathrm{s}, \mathrm{COO} t-\mathrm{Bu}), 2.49\left(2 \mathrm{H}, \mathrm{d}, J=6.4 \mathrm{~Hz}, 2-\mathrm{H}_{2}\right), 3.63$ and 3.78 (each $1 \mathrm{H}, \mathrm{dd}$, $J=13.6,6.7 \mathrm{~Hz}$ and $\left.13.5,6.6 \mathrm{~Hz}, 4-\mathrm{H}_{2}\right), 4.30-4.38(1 \mathrm{H}, \mathrm{m}, 3-\mathrm{H}), 9.19(1 \mathrm{H}$, $\mathrm{s}, \mathrm{CHO}), 9.28-10.12(1 \mathrm{H}, \mathrm{br}, \mathrm{COOH}) ;{ }^{13} \mathrm{C}-\mathrm{NMR}\left(\mathrm{CDCl}_{3}\right) \delta:-5.10$ and -4.59 (each s), $17.83(\mathrm{~s}), 25.68(\mathrm{q}), 28.06(\mathrm{q}), 40.79(\mathrm{t}), 45.53(\mathrm{t}), 66.59(\mathrm{~d})$, 84.53 (s), 152.33 (s), 163.32 (d), 176.54 (s); IR (KBr) cm ${ }^{-1}: 3174(\mathrm{OH})$ $1741,1712,1697(\mathrm{C}=\mathrm{O})$; MS (FAB) $m / z: 362\left(\mathrm{MH}^{+}\right)$; HR-MS (FAB) $m / z$ : 362.2002 (Calcd for $\left.\mathrm{C}_{16} \mathrm{H}_{32} \mathrm{NO}_{6} \mathrm{Si}: 362.1999\right) ;[\alpha]_{\mathrm{D}}^{26}-3.5\left(c=1.02, \mathrm{CHCl}_{3}\right)$.

(3R)-4-Amino-3-hydroxybutyric Acid (13) A mixture of the imide (12a, $1 \mathrm{mmol})$ in $2 \mathrm{M} \mathrm{HCl}(4 \mathrm{ml})$ and $\mathrm{AcOH}(4 \mathrm{ml})$ was heated at $60^{\circ} \mathrm{C}$ under argon atmosphere for $12 \mathrm{~h}$, and concentrated in vacuo. The obtained residue was desalted by ion-exchange chromatography on a Dowex $50 \mathrm{~W} \times 8(50$ 100 mesh, $38 \mathrm{ml}, \mathrm{H}^{+}$form) column with $2 \% \mathrm{NH}_{4} \mathrm{OH}$. Concentration of elute to dryness to give the crude $\omega$-acid, which was recrystallized from water-EtOH. $96 \mathrm{mg}\left(80 \%\right.$ from 12a); colorless prisms, $\mathrm{mp} 211^{\circ} \mathrm{C}\left(\right.$ lit. ${ }^{28)} \mathrm{mp}$ $\left.211-213^{\circ} \mathrm{C}\right) ;{ }^{1} \mathrm{H}-\mathrm{NMR}\left(\mathrm{D}_{2} \mathrm{O}\right) \delta: 2.38\left(2 \mathrm{H}, \mathrm{d}, J=6.5 \mathrm{~Hz}, 2-\mathrm{H}_{2}\right), 2.91$ and 3.12 (each $1 \mathrm{H}, \mathrm{dd}, J=13.1,9.4 \mathrm{~Hz}$ and $\left.13.1,3.2 \mathrm{~Hz}, 4-\mathrm{H}_{2}\right), 4.11-4.21(1 \mathrm{H}$, m, 3-H); ${ }^{13} \mathrm{C}-\mathrm{NMR}\left(\mathrm{D}_{2} \mathrm{O}\right) \delta: 43.19(\mathrm{t}), 44.99(\mathrm{t}), 66.39$ (d), $179.42(\mathrm{~s}) ; \mathrm{IR}$ $(\mathrm{KBr}) \mathrm{cm}^{-1}: 3413(\mathrm{OH}, \mathrm{NH}), 1577(\mathrm{C}=\mathrm{O})$; MS (FAB) $\mathrm{m} / z: 120\left(\mathrm{MH}^{+}\right)$; $[\alpha]_{\mathrm{D}}^{25}-21.1\left(c=0.79, \mathrm{H}_{2} \mathrm{O}\right)\left(\right.$ lit. $\left.^{28)}[\alpha]_{\mathrm{D}}^{28}-20.7\left(c=1.0, \mathrm{H}_{2} \mathrm{O}\right)\right) .102 \mathrm{mg}(85 \%$ from 12b).

Acknowledgements This work was partially supported by The Specific Research Found of Hokuriku University (2007).

\section{References and Notes}

1) Lee D. G., van den Engh M., "Oxidation in Organic Chemistry," ed. by Trahanovsky W. S., Academic Press, New York, 1973.

2) Plieker B., Synthesis, 2005, 2453-2472 (2005).

3) Sheehan J. C., Tulis R. W., J. Org. Chem., 39, 2264-2267 (1974).

4) Carlsen P. H. J., Katsuki T., Martin V. S., Sharpless K. B., J. Org. Chem., 46, 3936-3938 (1981).

5) Desai M. C., Chawla H. P. S., Dev S., Tetrahedron, 38, 379-382 (1982).

6) Torii S., Inokuchi T., Kondo K., J. Org. Chem., 50, 4980- 4982 (1985).

7) Yoshifuji S., Arakawa Y., Nitta Y., Chem. Pharm. Bull., 33, 50425047 (1985).

8) Yoshifuji S., Tanaka K., Kawai T., Nitta Y., Chem. Pharm. Bull., 33, 5515-5521 (1985)

9) Yoshifuji S., Tanaka K., Kawai T., Nitta Y., Chem. Pharm. Bull., 34, 3873-3878 (1986)

10) Yoshifuji S., Tanaka K., Nitta Y., Chem. Pharm. Bull., 33, 1749-1751 (1985).

11) Tanaka K., Yoshifuji S., Nitta Y., Chem. Pharm. Bull., 35, 364-369 (1987).

12) Yoshifuji S., Tanaka K., Nitta Y., Chem. Pharm. Bull., 35, 2994-3001 (1987).

13) Yoshifuji S., Matsumoto H., Tanaka K., Nitta Y., Tetrahedron Lett., 21 2963-2964 (1980)

14) Tanaka K., Yoshifuji S., Nitta Y., Chem. Pharm. Bull., 34, 3879-3884 (1986).

15) Kaname M., Yoshifuji S., Tetrahedron Lett., 33, 8103-8104 (1992).

16) Yoshifuji S., Kaname M., Chem. Pharm. Bull., 43, 1302-1306 (1995).

17) Yoshifuji S., Kaname M., Chem. Pharm. Bull., 43, 1617-1620 (1995).

18) Kaname M., Yoshifuji S., Sashida H., Tetrahedron Lett., 49, 2786$2788(2008)$

19) Yu J., Truc V., Riebel P., Hierl E., Mudryk B., Tetrahedron Lett., 46 4011-4013 (2005)

20) Oliveira D. F., Miranda P. C. M. L., Correia C. R. D., J. Org. Chem., 64, 6646-6652 (1999)

21) Schumacher K. K., Jiang J., Joullié M. M., Tetrahedron Asymmetry, 9, 47-53 (1998).

22) Dormoy J. R., Synthesis, 1982, 753-756 (1982).

23) Rossen K., Kolarovič A., Baskakov D., Kiesel M., Tetrahedron Lett., 45, 3023-3025 (2004)

24) Ferrari R., Di Mauro S., Sherwood G., "L-Carnitine and its Role in Medicine: from Function to Therapy," Academic, San Diego, 1992

25) De Simone C., Famularo G., "Carnitine Today," Springer Verlag, Heidelberg, 1997.

26) Jain R. P., Williams R. M., Tetrahedron, 57, 6505-6509 (2001) and references cited therein

27) Irie T., Ikota N., Fukushi K., Japan Patent 002637 (2001) [Chem. Abstr., 18, 945 (2001)].

28) Kamal A., Ramesh Khanna G. B., Krishnaji T., Ramu R., Tetrahedron Asymmetry, 17, 1281-1289 (2006). 